\title{
3
}

\section{UTILIZACIÓN DE NEUMÁTICOS USADOS COMO AGREGADO EN EL HORMIGÓN: CASO PROVINCIA DE SANTA ELENA, ECUADOR.}

Armando Saltos, Juan Garcés, Linda Ordoñez, Miguel Suárez, Humberto Guerrero. 


\title{
Utilización de neumáticos usados como agregado en el hormigón: caso provincia de Santa Elena, Ecuador
}

\section{Using tires used as added in the concrete: case province of Santa Elena, Ecuador}

\author{
Armando Saltos ${ }^{1}$, Juan Garcés², Humberto Guerrero ${ }^{1}$ Linda Ordoñez $^{2}$, Miguel Suarez ${ }^{2}$, \\ ${ }^{1}$ Universidad de Guayaquil UG - Facultad de Ciencias Matemáticas y Físicas \\ ${ }^{2}$ Universidad Península de Santa Elena UPSE - Facultad de Ciencias de Ingeniería \\ armandosaltos@hotmail.com
}

\begin{abstract}
Resumen
El proyecto encontró un diseño de hormigón con partículas provenientes de neumáticos usados, que después de un proceso de selección, trituración controlada, una dosificación acorde con los parámetros de las normas internacionales de diseño de hormigón del Instituto Americano del Concreto (ACI), la Norma Ecuatoriana de Construcción (NEC), la incorporación de hasta $10 \%$ de caucho triturado como sustituto parcial de agregados gruesos en el diseño de hormigón, se obtienen resultados favorables y de buena resistencia lo que hace que se pueda utilizar cumpliendo todas las normas ecuatorianas de la construcción. También se realizó un ensayo con un $10 \%$ de caucho triturado incorporado como sustituto parcial del agregado fino, resultando ser aún más apropiado para fines constructivos y de elaboración de hormigón. En el presente trabajo de investigación se realiza los ensayos como mezclas separadas. Se efectuaron pruebas de vigas con incorporación de agregados gruesos de hasta $15 \%$ cumpliendo las normas, pero no se ejecutó una serie completa.
\end{abstract}

Palabras clave: neumático, agregado, hormigón, resistencia.

\begin{abstract}
The project found a concrete design with particles from used tires, which after a selection process, controlled grinding, a dosage in accordance with the parameters of the American Concrete Institute (ICA) international standards of concrete design, Standard (NEC), the incorporation of up to $10 \%$ of tired rubber as a partial substitute of coarse aggregates in the concrete design, gives favorable results and good resistance which makes it possible to use all the Ecuadorian standards of the building. A test with $10 \%$ of crushed rubber incorporated as a partial substitute of the fine aggregate was also performed, making it even more suitable for construction and concrete processing purposes. In the present research work the tests are performed as separate mixtures. Beams were tested with incorporation of coarse aggregates of up to $15 \%$ complying with the standards, but a complete series was not performed.
\end{abstract}

Keywords: Tire, aggregate, concrete, resistance. 


\section{Introducción}

\subsection{Antecedentes}

La provincia de Santa Elena se encuentra ubicada en la región costa, limita al norte con la provincia de Manabí, al este con la provincia del Guayas y al sur y oeste con el Océano Pacífico.

El número de habitantes al censo del 2010, es de 308693 divididos en los tres cantones, donde la mayoría de estas personas corresponden al área rural, la población predominante es la mestiza y la edad promedio es de 27 años.

La provincia de Santa Elena tiene una superficie de $6050 \mathrm{Km}^{2}$. Las ciudades más importantes de la provincia son las cabeceras cantonales, así tenemos Santa Elena, La Libertad y Salinas, las cuales se comunican entre sí; $y$, con Guayaquil mediante una carretera de primer orden, asfaltada y de doble vía, la misma que se prolonga desde Santa Elena, hacia el Norte a lo largo de la Costa, hasta la provincia de Manabí.

Las llantas de desecho son consideradas un foco de riesgo ambiental y sanitario a nivel mundial, por el riesgo de un incendio o por quedar simple y sencillamente apiladas por años ya que se estiman que tardan 500 años en degradarse (https://prezi.com/lv0_q2nkfh_d/reciclaje-de llantas/.), generando riesgo de que se incrementen las emisiones a la atmósfera de diversas sustancias peligrosas.

Ecuador desecha al año, según el INVEC [4], cerca de 2,4 millones de neumáticos de diversos tipos, lo que equivale a 55.000 toneladas. Un menor porcentaje de ellos son reutilizados para el reencauchado, principalmente en vehículos de carga pesada, pero la gran mayoría es incinerada o depositada en basureros a cielo abierto, lo que supone una amenaza contra el medio ambiente y riesgos.

La ineficacia en la creación y aplicación de ordenanzas que regulen la disposición final de los neumáticos usados, siendo uno de los desperdicios más difíciles de manejar, que al terminar su ciclo de vida útil son eliminados; en el Ecuador "sólo $30 \%$ de los municipios tienen rellenos sanitarios", de los cuales no muchos tienen un lugar adecuado para la disposición de dichos desperdicios que afectan a la salud.
La acumulación de neumáticos a la intemperie representa un importante inconveniente para la salud, ya que en su interior se acumula agua proveniente de la lluvia, lo que se convierte en un 'foco' de reproducción de mosquitos que pueden causar enfermedades.

Otra repercusión del inadecuado desecho de estos elementos es que existen personas que compran las llantas usadas para el comercio del reencauche, pero hay quienes para evitar la acumulación prefieren quemarlas, con lo cual polucionan el aire.

La creación del diseño de hormigón con partículas provenientes de neumáticos, representó un material que, aunque puede ser hasta 20 por ciento más costoso, es altamente rentable, dado a que su duración puede ser cinco veces mayor.

\subsection{Objetivo General}

Minimizar el impacto ambiental producido por el mal manejo de neumáticos usados de los distintos sectores de transporte existentes en la provincia de Santa Elena y servir de ejemplo aplicado a todas las provincias del Ecuador.

\subsection{Objetivos específicos}

Implementar un diseño de hormigón compuesto con partículas provenientes de neumáticos usados del sector de transporte público y privado.

Socializar los resultados del estudio sobre los niveles de contaminación sobre la emanación de gases a lo que están expuestos los habitantes de la provincia de Santa Elena, con las autoridades de control ambiental, gobiernos seccionales, gubernamentales y dirigentes de las comunidades.

Evaluar los resultados de la mezcla de residuos granulados de llantas en el hormigón con aditivo de adherencia.

\subsection{Metodología}

Se adicionó un líquido adherente en la mezcla para mejorar la calidad de la mezcla.

Luego de análisis de la granulometría se estableció lo siguiente:

a) Diseño de hormigón (con materiales pétreos) para la elaboración de probetas para ensayos. 
b) Se adicionó un líquido adherente en la mezcla para mejorar la calidad de la mezcla.

c) Selección de porcentajes óptimos de cantidades de neumáticos triturados como agregados, para la elaboración de probetas, según diseños.

d) Ensayos de resistencias de las probetas de hormigón.

e) Tabulación de resultados de resistencias de las probetas.

f) Evaluación y análisis de los resultados.

g) Propuesta y selección de diseño óptimo con agregado de neumático triturado.

h) Conclusiones

\section{Materiales de Diseño}

\subsection{Información general}

Para un diseño de hormigón es necesario obtener una mezcla que posea determinadas propiedades tanto en estado fresco como endurecido, y que represente el menor costo de producción posible.

El hormigón, es un material de construcción constituido básicamente por rocas (Áridos), de tamaño máximo limitado, que cumplen ciertas condiciones en cuanto a sus características mecánicas, químicas y granulométricas, unidas entre sí por una pasta aglomerante hidráulica formada por un conglomerante (cemento) y agua. A este material básico y en el momento de su amasado, pueden añadírsele otros productos o materiales para mejorar algunas características determinadas (Adiciones y aditivos). A partir de este concepto se pueden añadir residuos triturados de neumáticos, que con una dosificación apropiada esté dentro de los rangos permisibles de resistencia para un diseño de hormigón.

\subsection{CARACTERÍSTICAS}

a. CEMENTO:

El cemento es el material ligante de los diferentes componentes del hormigón. El cemento para hormigones estructurales debe ser Portland, existiendo varios tipos; el tipo GU es el cemento a emplear en el diseño de hormigón con residuos triturados de llantas usadas.

\section{b. AGREGADOS:}

El agregado es el material granular, generalmente inerte, resultante de la desintegración natural y/o desgaste de rocas, o que se obtiene mediante la trituración de ellas, de escorias siderúrgicas convenientemente preparadas para tal fin o de otros materiales suficientemente duros, que permiten obtener partículas de forma y tamaños estables, destinadas a ser empleadas en hormigones (IRAM 1627:1997).

La importancia del uso del tipo y de la calidad correcta del agregado (árido) no se puede subestimar. Los agregados fino y grueso ocupan cerca del $60 \%$ al $75 \%$ del volumen del hormigón (70 \% a $85 \%$ de la masa) e influyen fuertemente en las propiedades, tanto en estado fresco como endurecido, en las proporciones de la mezcla y en la economía del hormigón. ${ }^{[2]}$

De acuerdo al tamaño de las partículas, los agregados se clasifican en agregados gruesos (tamaño mayor a $5 \mathrm{~mm}$ ) y agregados finos (tamaño entre $0.07 \mathrm{~mm}$ y $5 \mathrm{~mm}$ ). Una buena graduación de los agregados da lugar a hormigones de mejores características y más económicos. ${ }^{[3]}$

En la presente investigación se utilizó agregados artificiales como el caucho mezclado con los otros agregados produciéndose una mezcla de materiales.

La piedra empleada en esta investigación como agregado grueso es la extraída de la cantera Huayco, Guayaquil, tamaño $3 / 4$ " y tamaño $1 / 2$ ".

La arena empleada como agregado fino es de río de la provincia Guayas.

El agregado adicional, en este caso son los residuos triturados de neumáticos usados, provenientes de la recolección y clasificación previa.

Se quitó previamente toda impureza posible que se encuentre en el neumático antes de ser triturado para que no afecte al diseño de hormigón.

\section{c. AGUA}

El agua utilizada en el hormigón es potable y libre de impurezas de la red municipal local. No se utilizó agua de mar. 


\section{Propiedades de los neumáticos: ${ }^{[2]}$}

Amortiguación. - Es la capacidad de adaptarse a las irregularidades del terreno, y de absorber elásticamente los choques producidos por obstáculos pequeños.

Flexibilidad. - Debido a su propia constitución, los neumáticos se deforman con la aplicación de fuerzas exteriores: se denomina flexibilidad a la facilidad con que produce la deformación y su capacidad de recuperación.

Capacidad de carga. - Es el peso máximo que puede soportar un neumático, depende de sus características constructivas, de la presión de inflado, de la velocidad de vehículo, y de la clase de terreno por el que circule.

\section{Ensayos de los materiales.}

\subsection{Generalidades.}

Se denomina ensayos de materiales a toda prueba que tiene como fin determinar las propiedades mecánicas y químicas de un material, esto es, para comprobar si cumple o no con los estándares establecidos de normas vigentes, para verificar si es factible o no su empleo.

\subsection{Granulometría.}

El análisis granulométrico de los agregados consistió en separar y clasificar por tamaños los granos que lo componen con el fin de observar si se cumple especificaciones en las normas vigentes.

De acuerdo a los ensayos realizados, el polvo de caucho presenta un tamaño prácticamente uniforme, por lo tanto, no es necesario calcular su módulo de finura.

Además, se obtuvo el módulo de finura de la arena de las diferentes muestras, ver tabla 3.3:

Tabla 3.1.- Módulos de Finura de la Arena de Río utilizadas en esta investigación.

\begin{tabular}{|c|c|c|}
\hline $\begin{array}{c}\text { MUESTR } \\
\text { A }\end{array}$ & DESCRIPCIÓN & $\begin{array}{c}\text { MÓDUL } \\
\text { O DE } \\
\text { FINURA }\end{array}$ \\
\hline 1 & $\begin{array}{c}\text { Agregado Fino (Arena de Rio, } \\
\text { Guayas) utilizado en los } \\
\text { hormigones con el 10\% de }\end{array}$ & 3,0 \\
\hline
\end{tabular}

\begin{tabular}{|c|c|c|}
\hline & $\begin{array}{c}\text { polvo de caucho como agregado } \\
\text { fino. }\end{array}$ & \\
\hline 2 & $\begin{array}{c}\text { Agregado Fino (Arena de Rio, } \\
\text { Guayas) utilizado en los } \\
\text { hormigones con el 15 \% de } \\
\text { polvo de caucho como agregado } \\
\text { fino. }\end{array}$ & 2,6 \\
\hline 3 & $\begin{array}{c}\text { Agregado Fino (Arena de Rio, } \\
\text { Guayas) utilizado en los } \\
\text { hormigones con el 15\% de } \\
\text { polvo de caucho como agregado } \\
\text { fino y en hormigones con el 10 } \\
\text { y 15 \% de partículas de caucho } \\
\text { como agregado grueso. }\end{array}$ & 2,4 \\
\hline
\end{tabular}

Fuente: autores

\section{ANÁLISIS GRANULOMÉTRICO DEL AGREGADO GRUESO.}

Este análisis se basa en hacer pasar el material granular por una serie de tamices normados, y consiste esencialmente en obtener los porcentajes en pesos retenidos en cada uno de los tamices empleados. Para esta investigación se realizaron los ensayos respectivos y cumpliendo las normativas vigentes.

\subsection{Densidad, relativa y absorción.}

En el presente trabajo de investigación se realizaron los ensayos de densidad de la mezcla de materiales tradicionales con el caucho incluido, los mismos que se utilizaron para los diseños para diversas dosificaciones. Estos ensayos determinaron la densidad relativa y porcentaje de absorción de los agregados a utilizar en el diseño de hormigón posterior. Se realizaron los ensayos de esta investigación de acuerdo a las normas ASTM C 127 y ASTM C 128.

\subsection{Peso volumétrico.}

El peso volumétrico de un agregado es la relación que existe entre el peso de un material y el volumen que este ocupa y se expresa en $\mathrm{Kg} / \mathrm{m}^{3}$. Existen dos tipos de pesos volumétricos, el varillado y el suelto. Se evaluaron los pesos volumétricos de las mezclas.

\subsection{Resumen de resultados de ensayos}

En la tabla 3.2 se presenta el resumen de resultados de los ensayos realizados a los materiales en la presente investigación. 
Tabla 3.2.-Resumen de resultados de ensayos de los materiales de la presente investigación.

\begin{tabular}{|c|c|c|c|}
\hline \multirow[b]{2}{*}{ ENSAYO } & \multicolumn{3}{|c|}{ VALOR O ESTADO } \\
\hline & $\begin{array}{c}\text { MUESTRA } \\
1\end{array}$ & $\begin{array}{c}\text { MUESTRA } \\
2\end{array}$ & $\begin{array}{c}\text { MUESTRA } \\
\mathbf{3}\end{array}$ \\
\hline \multicolumn{4}{|c|}{ AGREGADO GRUESO ( PIEDRA) } \\
\hline $\begin{array}{l}\text { Análisis } \\
\text { Granulométrico }\end{array}$ & $\begin{array}{c}\text { SI } \\
\text { CUMPLE }\end{array}$ & $\begin{array}{c}\text { SI } \\
\text { CUMPLE }\end{array}$ & $\begin{array}{c}\text { SI } \\
\text { CUMPLE }\end{array}$ \\
\hline $\begin{array}{l}\text { Peso } \\
\text { Volumétrico } \\
\text { Suelto }\end{array}$ & $1429 \mathrm{~kg} / \mathrm{m}^{3}$ & $1334 \mathrm{~kg} / \mathrm{m}^{3}$ & $1110 \mathrm{~kg} / \mathrm{m}^{3}$ \\
\hline Densidad & $2680 \mathrm{~kg} / \mathrm{m}^{3}$ & $1790 \mathrm{~kg} / \mathrm{m}^{3}$ & $2700 \mathrm{~kg} / \mathrm{m}^{3}$ \\
\hline Absorción & $1,73 \%$ & $1,98 \%$ & $2,20 \%$ \\
\hline \multicolumn{4}{|c|}{ AGREGADO FINO (ARENA) } \\
\hline $\begin{array}{l}\text { Análisis } \\
\text { Granulométrico }\end{array}$ & $\begin{array}{c}\text { SI } \\
\text { CUMPLE }\end{array}$ & $\begin{array}{c}\text { SI } \\
\text { CUMPLE }\end{array}$ & $\begin{array}{c}\text { SI } \\
\text { CUMPLE }\end{array}$ \\
\hline $\begin{array}{l}\text { Peso } \\
\text { Volumétrico }\end{array}$ & $1682 \mathrm{~kg} / \mathrm{m}^{3}$ & $1288 \mathrm{~kg} / \mathrm{m}^{3}$ & $1290 \mathrm{~kg} / \mathrm{m}^{3}$ \\
\hline Densidad & $2689 \mathrm{~kg} / \mathrm{m}^{3}$ & $2700 \mathrm{~kg} / \mathrm{m}^{3}$ & $2700 \mathrm{~kg} / \mathrm{m}^{3}$ \\
\hline Absorción & $3,95 \%$ & $3,95 \%$ & $1,24 \%$ \\
\hline \multicolumn{4}{|c|}{ AGREGADO ADICIONAL (NEUMÁTICO TRITURADO) } \\
\hline $\begin{array}{l}\text { Análisis } \\
\text { Granulométrico }\end{array}$ & $\begin{array}{c}\text { DIÁMETR } \\
\text { O DE } 1 \text { A } 3 \\
\text { mm } \\
\text { (AGREGA } \\
\text { DO FINO) }\end{array}$ & $\begin{array}{c}\text { DIÁMETR } \\
\text { O DE } 5 \text { A } \\
20 \mathrm{~mm} \\
\text { (AGREGA } \\
\text { DO } \\
\text { GRUESO) }\end{array}$ & - \\
\hline $\begin{array}{l}\text { Peso } \\
\text { Volumétrico }\end{array}$ & $381 \mathrm{~kg} / \mathrm{m}^{3}$ & - & - \\
\hline Densidad & $1000 \mathrm{~kg} / \mathrm{m}^{3}$ & $1000 \mathrm{~kg} / \mathrm{m}^{3}$ & - \\
\hline
\end{tabular}

Fuente: autores

\section{Diseño de hormigón con residuos de neumático}

\subsection{Pre diseño de mezcla típica (sin adición de residuos de neumático)}

En un diseño de mezcla, existen valores límites que pueden cubrir una extensa lista de propiedades; estos valores deben, entonces satisfacerse en los cálculos de diseño de mezclas y forman de hecho la base para la selección y dosificación de los componentes de mezcla.

El método empleado para la realización del prediseño y diseño de hormigón con residuos triturados de neumáticos usados, es el método propuesto por el ACI en la norma 211.1 - 70 (con ajustes de ACI-2008). a) Procedimiento de cálculo para el prediseño de la mezcla de hormigón simple (Pre-Diseño Patrón para mezclas con $10 \%$ de Caucho Triturado como agregado fino)

1) Los resultados obtenidos previamente de los ensayos de laboratorio de los materiales se presentan en la tabla 4.1, donde se detallan además las características que debe cumplir el prediseño a calcular.

Tabla 4.1.-Características del prediseño de hormigón:

\section{DATOS}

\begin{tabular}{|l|c|l|}
\hline $\begin{array}{l}\text { Resistencia característica } \\
\text { (fc })=\end{array}$ & 210 & $\mathrm{~kg} / \mathrm{cm}^{2}$ \\
\hline Asentamiento de Abraham= & 50 & $\mathrm{Mm}$ \\
\hline Tipo de cemento (C)= & NORMAL & \\
\hline $\begin{array}{l}\text { Tamaño máximo de Agregado } \\
\text { Grueso ( AG ) }=\end{array}$ & 25,4 & $\mathrm{Mm}$ \\
\hline $\begin{array}{l}\text { Densidad del Agregado Grueso } \\
\text { ( AG ) }\end{array}$ & 2680 & $\mathrm{~kg} / \mathrm{m}^{3}$ \\
\hline $\begin{array}{l}\text { Peso volumétrico Agregado } \\
\text { Grueso ( AG ) }\end{array}$ & 1429 & $\mathrm{Kg} / \mathrm{m}^{3}$ \\
\hline $\begin{array}{l}\text { Peso volumétrico Agregado } \\
\text { Grueso Varillado( AG ) }\end{array}$ & 1558 & $\mathrm{Kg} / \mathrm{m}^{3}$ \\
\hline $\begin{array}{l}\% \text { de absorción del Agregado } \\
\text { grueso (AG) }=\end{array}$ & $\%, 73$ & $\%$ \\
\hline $\begin{array}{l}\text { Módulo de finura de agregado } \\
\text { fino (AF) }=\end{array}$ & 2689 & $\mathrm{~kg} / \mathrm{m}^{3}$ \\
\hline $\begin{array}{l}\text { Peso volumétrico del agregado } \\
\text { fino= }\end{array}$ & $\%, 95$ & $\%$ \\
\hline $\begin{array}{l}\text { Densidad del agregado fino } \\
\text { (AF)= }\end{array}$ & 1682 & $\mathrm{Kg} / \mathrm{m}^{3}$ \\
\hline $\begin{array}{l}\% \text { de absorción del Agregado } \\
\text { fino (AF) }\end{array}$ & Futores \\
\hline
\end{tabular}

Se determinó la resistencia requerida del hormigón, de acuerdo la ecuación establecida por ACI cuando no hay datos para establecer la desviación estándar de la muestra (ver tabla 4.2), y al estar la resistencia especificada a la compresión en el rango entre 21 y $35 \mathrm{MPa}$, se selecciona la segunda fórmula:

$$
f^{\prime} c r=f^{\prime} c+8,3
$$

$\begin{array}{lrl}\text { Reemplazando } & \mathrm{f}^{\prime} \mathrm{c} ; \\ \mathrm{f}^{\prime} \mathrm{cr}= & 28,89 & \mathrm{Mpa} \\ \mathrm{f}^{\prime} \mathrm{cr}= & 294,6 & \mathrm{~kg} / \mathrm{cm}^{2}\end{array}$


Tabla 4.2.-Resistencia promedio a la compresión requerida cuando no hay datos disponibles para establecer una desviación estándar de la muestra.

Fuente: ACI 318, Tabla 5.3.2.2.

\begin{tabular}{|c|c|}
\hline $\begin{array}{c}\text { Resistencia } \\
\text { específica a la } \\
\text { compresión, Mpa }\end{array}$ & $\begin{array}{c}\text { Resistencia } \\
\text { promedio requerida a } \\
\text { la compresión, Mpa }\end{array}$ \\
\hline$f_{c}^{\prime}<21$ & $f_{c r}^{\prime}=f_{c}^{\prime}+7.0$ \\
\hline $21 \leq f_{c}^{\prime} \leq 35$ & $f_{c r}^{\prime}=f_{c}^{\prime}+8.3$ \\
\hline$f_{c}^{\prime}>35$ & $f_{c r}^{\prime}=1.10 f_{c}^{\prime}+5.0$ \\
\hline
\end{tabular}

Se calcula la cantidad de agua requerida y el porcentaje de aire atrapado según la tabla 4.3

Tabla 4.3.-Cantidad aproximada de agua de mezclado para diferentes asentamientos y tamaño máximo de agregado.

\begin{tabular}{|c|c|c|c|c|}
\hline & \multicolumn{5}{|c|}{$\begin{array}{l}\text { CANTIDAD DE AGUA (Kg/m3 } \\
\text { de concreto para agregados de } \\
\text { tamaño máximo) }\end{array}$} \\
\hline ASENTAMIENTO & $10 \mathrm{~mm}$ & $12,5 \mathrm{~mm}$ & $20 \mathrm{~mm}$ & $25 \mathrm{~mm}$ \\
\hline $30-50$ & 205 & 200 & $\underline{\mathbf{1 8 5}}$ & 180 \\
\hline $80-100$ & 225 & 215 & 200 & 195 \\
\hline $150-180$ & 240 & 230 & 210 & 205 \\
\hline $\begin{array}{c}\text { Contenido de aire } \\
\text { atrapado (\%) }\end{array}$ & 3 & 2.5 & $\underline{\mathbf{2}}$ & 1,5 \\
\hline \multicolumn{7}{|c|}{ Fuente: Marcelo Romo, 2008. } \\
\hline
\end{tabular}

Considerando un asentamiento de $50 \mathrm{~mm}$ y el tamaño de $19 \mathrm{~mm}$ del agregado grueso, se tiene que:

Cantidad de agua por metro cúbico: $185 \mathrm{~kg}$.

Porcentaje de volumen de aire atrapado: $2 \%$

Se determinó la relación agua/cemento a partir de la gráfica 4.1, con la resistencia media (fcr) calculada anteriormente, de donde se obtiene que:

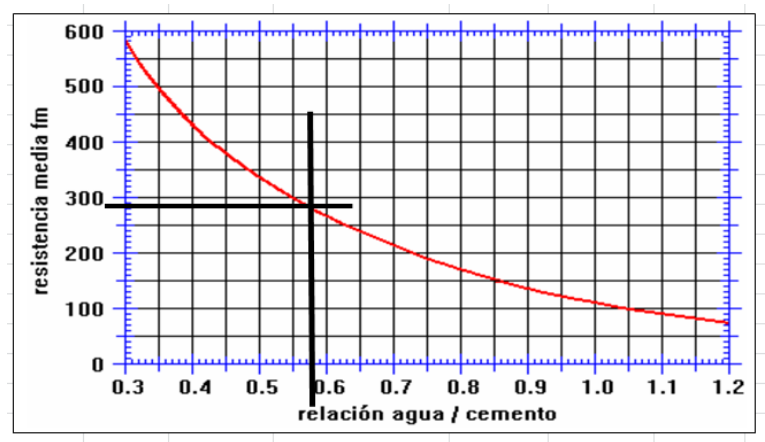

Relación Agua/Cemento = 0,471
Gráfica 4.1.-. Curva de la resistencia media del hormigón como función de la relación agua/cemento

Fuente: Marcelo Romo, 2008

A partir del peso del agua y la relación agua/cemento se obtiene el peso de cemento a emplear en $1 \mathrm{~m} 3$.

$$
\begin{aligned}
& \text { Peso de Cemento }=\frac{\text { peso del agua }}{\text { relación } \frac{\mathrm{a}}{\mathrm{c}}} \\
& \text { Peso de Cemento }=320,07 \mathrm{~kg}
\end{aligned}
$$

Posteriormente se obtiene el volumen aparente del agregado grueso por metro cubico de hormigón, tomando en cuenta el tamaño máximo del agregado de $25 \mathrm{~mm}$ y el módulo de finura del arena 2,4 , a partir de la tabla 4.4

Tabla 4.4.-Volumen aparente de agregado grueso por metro cubico de hormigón.

\begin{tabular}{|c|c|c|c|c|}
\hline $\begin{array}{c}\text { Tamaño } \\
\text { máximo } \\
\text { del } \\
\text { agregado }\end{array}$ & \multicolumn{4}{|c|}{$\begin{array}{c}\text { Volumen de agregado grueso } \\
\text { compactado con varilla por volumen } \\
\text { de concreto para módulo de finura de } \\
\text { la arena }\end{array}$} \\
\hline $\mathrm{mm}$ & 2.4 & 2.6 & 2.8 & 3 \\
\hline 10 & 0,5 & 0,48 & 0,46 & 0,44 \\
\hline 12,5 & 0,59 & 0,57 & 0,55 & 0,53 \\
\hline 20 & $\underline{\mathbf{0 , 6 6}}$ & 0,64 & 0,62 & 0,6 \\
\hline 25 & 0,71 & 0,69 & 0,67 & 0,65 \\
\hline 40 & 0,75 & 0,73 & 0,71 & 0,69 \\
\hline 50 & 0,78 & 0,76 & 0,74 & 0.72 \\
\hline 70 & 0,82 & 0,8 & 0,78 & 0,76 \\
\hline 150 & 0,87 & 0,85 & 0,83 & 0,81 \\
\hline
\end{tabular}

Vol. $A G=0,66 \mathrm{~m}^{3}$

Posteriormente se obtiene el peso del agregado grueso a través de la siguiente fórmula:

Peso del $A G=($ volumen aparente de $A G) /($ peso volumétrico del $A G)$

Peso del $A G=1028,28 \mathrm{~kg}$

Luego se calculó el volumen de cemento, agua, agregado grueso, mediante la siguiente ecuación:

Volumen $=$ peso $/$ densidad

Entonces:

Volumen Cemento $=0,102 \mathrm{~m}^{3}$

Volumen de Agua $=0,185 \mathrm{~m}^{3}$

Volumen Agregado Grueso: 0,3837 $\mathrm{m}^{3}$ 
Se calcula el aire atrapado (AA):

Volumen de aire atrapado $=\%$ aire atrapado $/ 1 \mathrm{~m}^{3}$

Volumen aire atrapado $=0,020 \mathrm{~m}^{3}$

De acuerdo a los datos de absorción de los agregados se realiza la corrección de la cantidad de agua necesaria en la mezcla.

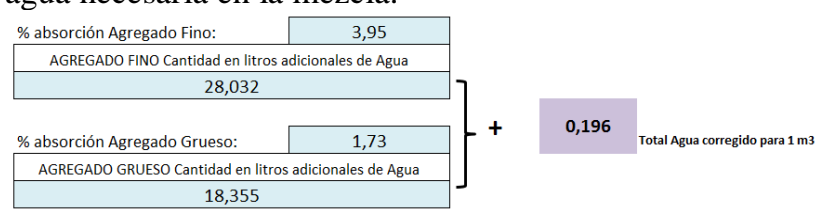

Se calcula el volumen de agregado fino mediante la fórmula:

Vol. agregado fino= $1 \mathrm{~m} 3$ - Vol. Cem. - Vol. AG Vol. AA - Vol. Agua - Vol. AN

Vol. agregado fino $=0,299 \mathrm{~m}^{3}$

Se calcula el peso del agregado fino:

Peso agregado fino= Volumen agregado fino $x$ densidad agregado fino

Peso agregado fino $=804,54 \mathrm{~kg}$

A continuación, se muestra la tabla con el resumen de la dosificación.

Tabla 4.5.-Dosificación por metro cubico de hormigón, en volumen y peso.

\begin{tabular}{|c|c|c|}
\hline Material & $\mathbf{m 3}$ & $\mathbf{~ K g}$ \\
\hline CEMENTO & 0,102 & 320 \\
\hline AF & 0,299 & 805 \\
\hline AG & 0,384 & 1028 \\
\hline AGUA & 0,196 & 196 \\
\hline AA & 0,020 & 0 \\
\hline HORMIGON FRESCO & 1,000 & 2348,40 \\
\hline \multicolumn{3}{|c|}{ Fuente: Autores }
\end{tabular}

Entonces la dosificación corregida por saco de cemento, es:

Tabla 4.6.-Dosificación corregida por metro cúbico de hormigón, en volumen y peso.

\begin{tabular}{|c|c|l|}
\hline Material & Cantidad & Unidad \\
\hline CEMENTO & 50,00 & $\mathrm{Kg}$ \\
\hline AF & 114,74 & $\mathrm{Kg}$ \\
\hline AG & 171,54 & Kg \\
\hline AGUA & 31 & Litros \\
\hline
\end{tabular}

Fuente: Autores

b) Procedimiento de cálculo para el prediseño de la mezcla de hormigón, con un $10 \%$ de partículas de neumáticos como agregado fino, utilizando las Muestras 1 de piedra y arena.

Los pasos del 1 al 15 del procedimiento "a" se repiten, se procede a calcular la cantidad de caucho que reemplazaría al $10 \%$ de arena como agregado fino, quedando de la siguiente manera, tabla 4.8.

Tabla 4.7.-Volumenes y pesos (V AN y P AN) de caucho triturado en relación a la arena.

\begin{tabular}{|c|c|c|}
\hline $\begin{array}{c}\text { \% AN CON } \\
\text { RELACIÓN AF }\end{array}$ & VAN & $\begin{array}{c}\text { V RESIDUAL } \\
\text { AF }\end{array}$ \\
\hline$\%$ & M3 & M3 \\
\hline 10 & 0,0273 & 0,246 \\
\hline
\end{tabular}

\begin{tabular}{|c|c|c|}
\hline $\begin{array}{c}\text { \% AN CON } \\
\text { RELACIÓN AF }\end{array}$ & PAN & $\begin{array}{c}\text { PRESIDUAL } \\
\text { AF }\end{array}$ \\
\hline$\%$ & $\mathrm{Kg}$ & $\mathrm{Kg}$ \\
\hline 10 & 73,45 & 661,06 \\
\hline
\end{tabular}

Fuente: Autores

De la tabla 4.7, el Peso de agregado neumático (AN) aparente relacionado con el $10 \%$ del peso de la arena (AF) se lo divide entre la densidad de la arena para poder sacar el volumen que este porcentaje ocupa, así:

Densidad $=\quad$ peso/volumen $\quad=>$ Volumen $=\quad 0,0273 \mathrm{~m} 3$

Y multiplicando el valor de $0,0273 \mathrm{~m} 3$ por la densidad del caucho, obtenemos el peso del caucho que reemplazaría a $73,45 \mathrm{~kg}$ del peso de la arena:

Peso $=27,32 \quad K G$

Entonces se necesitan 27,32 $\mathrm{Kg}$ de polvo de caucho para reemplazar $73,45 \mathrm{Kg}$ de arena equivalente al $10 \%$.

En la tabla 4.8 se muestran los valores de los agregados para la dosificación para $1 \mathrm{~m} 3$ de hormigón con caucho triturado.

Tabla 4.8.-Dosificación por metro cúbico de hormigón, con el $10 \%$ de partículas de caucho triturado como agregado fino, en volumen y peso.

\begin{tabular}{|c|c|c|}
\hline Material & $\begin{array}{c}\text { Vol Neto } \\
\mathbf{~ m 3}\end{array}$ & $\begin{array}{c}\text { Peso } \\
\mathbf{~ K g}\end{array}$ \\
\hline CEMENTO & 0,102 & 320,07 \\
\hline AF & 0,246 & 661,058 \\
\hline AG & 0,410 & 1098,08 \\
\hline AN & 0,027 & 27,315 \\
\hline AGUA & 0,196 & 196 \\
\hline AA & 0,020 & 0 \\
\hline
\end{tabular}

Además, en la tabla 4.9 se presenta la dosificación por saco de cemento. 
Tabla 4.9.-Dosificación por saco de cemento, con el $10 \%$ de partículas de caucho triturado como agregado fino, en volumen y peso.

\begin{tabular}{|c|c|l|}
\hline Material & Cantidad & Unidad \\
\hline CEMENTO & 50,00 & $\mathrm{Kg}$ \\
\hline $\mathrm{AF}$ & 103,27 & $\mathrm{Kg}$ \\
\hline $\mathrm{AG}$ & 171,54 & $\mathrm{Kg}$ \\
\hline $\mathrm{AN}$ & 4,27 & $\mathrm{Kg}$ \\
\hline AGUA & 30,54 & Litros \\
\hline \multicolumn{3}{|c}{ Fuente: Autores }
\end{tabular}

\subsection{Propiedades del hormigón endurecido: ensayos.}

\subsubsection{Ensayo de resistencia a la compresión simple}

El valor de la resistencia a la compresión simple de un espécimen de hormigón es su característica mecánica principal, dada la importancia que representa esta propiedad, dentro de una estructura típica de hormigón reforzado, en términos de esfuerzo se la expresa generalmente en $\mathrm{kg} / \mathrm{cm} 2$ y con alguna frecuencia $\mathrm{MPa}$. La equivalencia que hay entre los dos es que $1 \mathrm{MPa}$ es igual a 10,196 $\mathrm{kg} / \mathrm{cm} 2 .^{[5]}$

\subsection{Evaluación de resultados.}

\section{HORMIGÓN ELABORADO CON 10\% DE CAUCHO TRITURADO INCORPORADO COMO AGREGADO FINO}

En la tabla 5a se muestran los resultados del ensayo a compresión simple de los especímenes cilíndricos a los 28 días de su fundición. Estos resultados se encuentran en $\mathrm{kg} / \mathrm{cm} 2$.

El resultado de la muestra patrón indica que alcanzó una resistencia máxima (f'c) a los 28 días de $224 \mathrm{~kg} / \mathrm{cm} 2$, lo que representa una eficiencia del $107 \%$ con respecto al f'c de diseño que es de $210 \mathrm{~kg} / \mathrm{cm} 2$, resultado aceptable para esta investigación.

Tabla 5a.-Resistencia a los 28 días en cilindros con $10 \%$ de caucho triturado incorporado como agregado fino.

\begin{tabular}{|c|c|c|}
\hline $\begin{array}{c}\# \\
\text { ENSAYO }\end{array}$ & $\begin{array}{c}\% \\
\text { RESISTENCIA } \\
\text { KG/CM2 }\end{array}$ & $\begin{array}{c}\text { Eficiencia } \\
\text { alcanzado }\end{array}$ \\
\hline
\end{tabular}

\begin{tabular}{|c|c|r|} 
PATRÓN & 224,77 & 107,03 \\
\hline 1 & 200,95 & 95,69 \\
\hline 2 & 188,15 & 89,59 \\
\hline 3 & 189,68 & 90,32 \\
\hline 4 & 188,06 & 89,55 \\
\hline 5 & 202,06 & 96,22 \\
\hline 6 & 221,12 & 105,30 \\
\hline 7 & 217,21 & 103,43 \\
\hline 8 & 182,07 & 86,70 \\
\hline 9 & 187,43 & 89,25 \\
\hline 10 & 182,07 & 86,70 \\
\hline 11 & 217,34 & 103,49 \\
\hline 12 & 211,80 & 100,86 \\
\hline 13 & 244,16 & 116,27 \\
\hline 14 & 221,62 & 105,53 \\
\hline 15 & 178,86 & 85,17 \\
\hline
\end{tabular}

Fuente: Autores.

El promedio alcanzado de las pruebas de resistencia a la compresión de los resultados de especímenes con $10 \%$ de caucho triturado incorporado como agregado fino es de $191.71 \mathrm{~kg} / \mathrm{cm}^{2}$, lo que representa un $91,29 \%$ de la resistencia de diseño f'c que es de $210 \mathrm{~kg} / \mathrm{cm} 2$.

Tomando en consideración estos resultados se obtiene que la resistencia disminuye en un $8,71 \%$ en relación a la resistencia de diseño. Lo cual es aceptable entre los parámetros de diseño; se debe considerar que no se utilizó ningún tipo de aditivo.

Para los ensayos realizados con un $10 \%$ de caucho incorporado como agregado fino se obtuvo la desviación estándar como se muestra en la tabla 5a1. La desviación estándar " $S$ " se obtuvo mediante promedios obtenidos de 15 ensayos realizados (promedio de resistencias de dos probetas), según lo indica Instituto Ecuatoriano del Cemento y del Concreto.

Tabla 5a1.-Desviación estándar para 10\% de caucho triturado como agregado fino.

\begin{tabular}{|r|c|c|c|c|c|}
\hline $\begin{array}{r}\mathrm{N}^{\circ} \\
\text { ENSAYO }\end{array}$ & $\begin{array}{c}\text { RES. } \\
\text { DISE. } \\
\mathrm{Kg} / \mathrm{cm}^{2}\end{array}$ & $\begin{array}{c}\text { PROB 1 } \\
\mathrm{Kg} / \mathrm{cm}^{2}\end{array}$ & $\begin{array}{c}\text { PROB 2 } \\
\mathrm{Kg} / \mathrm{cm}^{2}\end{array}$ & $\begin{array}{c}\mathrm{Xi}(\mathrm{prom}) \\
\mathrm{Kg} / \mathrm{cm}^{2}\end{array}$ & $\mathrm{Xi}^{2}$ (prom) \\
\hline 1 & 210 & 198,83 & 203,08 & 200,96 & 40382,91 \\
\hline 2 & 210 & 203,54 & 172,76 & 188,15 & 35400,42 \\
\hline 3 & 210 & 196,51 & 182,84 & 189,68 & 35976,61 \\
\hline 4 & 210 & 188,88 & 187,24 & 188,06 & 35366,56 \\
\hline 5 & 210 & 234,43 & 169,69 & 202,06 & 40828,24 \\
\hline 6 & 210 & 227,77 & 214,47 & 221,12 & 48894,05 \\
\hline 7 & 210 & 218,89 & 215,52 & 217,21 & 47178,01 \\
\hline
\end{tabular}




\begin{tabular}{|c|c|c|c|c|c|}
\hline 8 & 210 & 186,2 & 177,94 & 182,07 & 33149,48 \\
\hline 9 & 210 & 147,65 & 227,2 & 187,43 & 35128,13 \\
\hline 10 & 210 & 186,2 & 177,94 & 182,07 & 33149,48 \\
\hline 11 & 210 & 228,83 & 205,85 & 217,34 & 47236,68 \\
\hline 12 & 210 & 217,03 & 206,58 & 211,81 & 44861,36 \\
\hline 13 & 210 & 243,52 & 244,81 & 244,17 & 59616,55 \\
\hline 14 & 210 & 226,21 & 217,03 & 221,62 & 49115,42 \\
\hline 15 & 210 & 185,92 & 171,79 & 178,86 & 31989,11 \\
\hline & \multicolumn{3}{|c|}{$\mathrm{N}^{\circ}$ ENSAYO $" n "=$} & 15,0 & \\
\hline & \multicolumn{3}{|c|}{$\begin{array}{c}\ddot{\mathrm{X}}=\begin{array}{c}\text { Promedio } \mathrm{Xi} \text { en } \mathrm{n} \\
\text { ensayos }\end{array} \\
\end{array}$} & 202 & \\
\hline & \multicolumn{3}{|c|}{$\Sigma X i^{2}=$} & 618273,0 & \\
\hline & \multicolumn{3}{|c|}{$\begin{array}{c}\text { Desviación Estándar (S) } \\
=\end{array}$} & 19,23 & \\
\hline
\end{tabular}

\begin{tabular}{|c|c|}
\hline $\begin{array}{l}\mathrm{S} \text { con factor de } \\
\text { modificación= }\end{array}$ & 22,31 \\
\hline $\begin{array}{l}\mathrm{S} \text { con factor de } \\
\text { modificación= }\end{array}$ & 2,19 \\
\hline
\end{tabular}

Fuente: Autores.

El factor $\mathrm{S}$ es 2.19 , por lo que este valor se utiliza para elaborar el diseño definitivo de hormigón para un $10 \%$ de caucho triturado incorporado como agregado fino. El diseño completo se encuentra en el Anexo 4 de este informe.

Tabla 5a2.-Diseño final con $10 \%$ de caucho triturado como agregado fino. En términos de volumen $(\mathrm{m} 3)$ y peso $(\mathrm{kg})$.

\begin{tabular}{|c|c|c|}
\hline Material & $\begin{array}{c}\text { Vol. } \\
\text { Neto } \\
\mathbf{m}^{\mathbf{3}}\end{array}$ & $\begin{array}{c}\text { Peso } \\
\mathbf{K g}\end{array}$ \\
\hline CEMENTO & 0,098 & 307,82 \\
\hline $\mathrm{AF}$ & 0,247 & 664,822 \\
\hline $\mathrm{AG}$ & 0,412 & 1104,33 \\
\hline $\mathrm{AN}$ & 0,027 & 27,471 \\
\hline AGUA & 0,196 & 196 \\
\hline AA & 0,020 & 0 \\
\hline
\end{tabular}

Fuente: Autor.

Tabla 5a3.-Diseño final con $10 \%$ de caucho triturado como agregado fino. Por saco de cemento de $50 \mathrm{~kg}$.

\begin{tabular}{|c|c|c|}
\hline Material & Cantidad & Unidad \\
\hline CEMENTO & 50,00 & $\mathrm{Kg}$ \\
\hline $\mathrm{AF}$ & 107,99 & $\mathrm{Kg}$ \\
\hline $\mathrm{AG}$ & 179,38 & $\mathrm{Kg}$ \\
\hline $\mathrm{AN}$ & 4,46 & $\mathrm{Kg}$ \\
\hline AGUA & 31,76 & Litros \\
\hline
\end{tabular}

Fuente: Autores.

A Saltos/J. Garcés/L. Ordoñez/M. Suárez/H. Guerrero.
La tabla 5a2 presenta la dosificación de los materiales para un metro cúbico de hormigón $\left(1 \mathrm{~m}^{3}\right)$, además su representación en peso, para un $10 \%$ de caucho triturado incorporado como agregado fino en la mezcla de hormigón. Mientras la tabla $5 \mathrm{a} 3$ presenta la dosificación final en peso (kg) para un saco de cemento de $50 \mathrm{~kg}$, que es la que se expende en este medio.

\section{HORMIGÓN ELABORADO CON 15\% DE CAUCHO TRITURADO INCORPORADO COMO AGREGADO FINO}

A continuación, se presenta en la tabla $5 b$ los resultados de las pruebas a compresión simple de especímenes con un $15 \%$ de caucho triturado incorporado como agregado fino. Además, se encuentra el resultado de la misma prueba del cilindro como muestra patrón.

Tabla 5b.-Resistencia a los 28 días en cilindros con $15 \%$ de caucho triturado incorporado como agregado fino.

\begin{tabular}{|c|c|c|}
\hline \# ENSAYO & $\begin{array}{c}\text { RESISTENCIA } \\
\mathrm{kg} / \mathrm{cm}^{2}\end{array}$ & \% EFICIENCIA \\
\hline PATRÓN & 257,46 & 122,60 \\
\hline 1 & 193,03 & 91,92 \\
\hline 2 & 166,72 & 79,39 \\
\hline 3 & 162,15 & 77,22 \\
\hline 4 & 165,29 & 78,71 \\
\hline 5 & 174,68 & 83,18 \\
\hline 6 & 147,95 & 70,45 \\
\hline 7 & 170,05 & 80,98 \\
\hline 8 & 183,11 & 87,20 \\
\hline 9 & 163,38 & 77,80 \\
\hline 10 & 145,44 & 69,26 \\
\hline 11 & 158,11 & 75,29 \\
\hline 12 & 161,88 & 77,08 \\
\hline 13 & 175,66 & 83,65 \\
\hline 14 & 148,90 & 70,90 \\
\hline 15 & 160,11 & 76,24 \\
\hline
\end{tabular}

Fuente: Autores.

El promedio de resistencia alcanzada a los 28 días de los 15 ensayos de los especímenes con un $15 \%$ de caucho triturado incorporado como agregado fino es de $153.39 \mathrm{~kg} / \mathrm{cm}^{2}$, lo que representa un $73,04 \%$ de eficiencia en relación al diseño patrón de 
referencia que es de $\mathrm{f}^{\prime} \mathrm{c}=210 \mathrm{~kg} / \mathrm{cm}^{2}$. El resultado de la prueba al espécimen tomado como muestra patrón es aceptable ya que es mayor a la de diseño $\left(f^{\prime} c=210 \mathrm{~kg} / \mathrm{cm}^{2}\right)$. De este resultado se obtiene que la resistencia alcanzada de los especímenes disminuyó en un $26.96 \%$ en relación a la muestra patrón. No se realizaron los 15 ensayos necesarios (promedio de 2 pruebas realizadas) para poder obtener la desviación estándar con el $15 \%$ de caucho triturado, por lo que no se obtuvo un diseño final para este porcentaje.

\section{HORMIGÓN ELABORADO CON 10\% DE CAUCHO TRITURADO INCORPORADO COMO AGREGADO GRUESO}

El promedio de resistencia alcanzada a los 28 días de los 15 ensayos de los especímenes con un $10 \%$ de caucho triturado incorporado como agregado grueso es de $153.13 \mathrm{~kg} / \mathrm{cm}^{2}$, lo que representa un $72.92 \%$ de eficiencia en relación al diseño patrón de referencia que es de $\mathrm{f}^{\prime} \mathrm{c}=210 \mathrm{~kg} / \mathrm{cm}^{2}$. La tabla $5 \mathrm{c}$ muestra los resultados de los ensayos realizados a los 28 días. Dado los resultados relativamente bajos, se realizaron ensayos a un promedio de 90 días, los cuales alcanzaron resistencias $f^{\prime} c=200 \mathrm{~kg} / \mathrm{cm}^{2}$, dando una eficiencia de $96 \%$ en relación al f'c de diseño que es de $210 \mathrm{~kg} / \mathrm{cm}^{2}$. Es decir, hubo un incremento del $23 \%$ de resistencia a la compresión.

Tabla 5c.-Resistencia a los 28 días en cilindros con $10 \%$ de caucho triturado incorporado como agregado grueso.

\begin{tabular}{|c|c|c|}
\hline \# ENSAYO & $\begin{array}{c}\text { RESISTENCIA } \\
\mathrm{kg} / \mathrm{cm}^{2}\end{array}$ & $\begin{array}{c}\% \\
\text { EFICIENCIA }\end{array}$ \\
\hline PATRON & 172,97 & 82,37 \\
\hline 1 & 193,03 & 91,92 \\
\hline 2 & 166,72 & 79,39 \\
\hline 3 & 190,96 & 90,93 \\
\hline 4 & 189,11 & 90,05 \\
\hline 5 & 163,85 & 78,02 \\
\hline 6 & 191,16 & 91,03 \\
\hline 7 & 192,94 & 91,88 \\
\hline 8 & 183,07 & 87,17 \\
\hline 9 & 161,92 & 77,11 \\
\hline 10 & 137,11 & 65,29 \\
\hline 11 & 138,60 & 66,00 \\
\hline 12 & 144,32 & 68,72 \\
\hline 13 & 143,41 & 68,29 \\
\hline 14 & 122,98 & 58,56 \\
\hline 15 & 121,25 & 57,74 \\
\hline \multicolumn{3}{|c|}{ Fuente: Autor. } \\
\hline
\end{tabular}

A Saltos/J. Garcés/L. Ordoñez/M. Suárez/H. Guerrero.
En los ensayos de resistencia de hormigones con material de caucho grueso se utilizó un aditivo el SIKA TOP 77 de la empresa Sika, aditivo que se lo incorporó porque en las primeras pruebas evaluadas sin aditivo el caucho no se adhería bien y con la incorporación del aditivo aumentaba la adherencia y por ende la resistencia, en los ensayos se analizó con varios ensayos de adherencia que con la proporción de 20 a 1 relación agua aditivo los resultados resultaron exitosos.

Existen variaciones en algunas probetas ensayadas, dado que este estudio se basa en resistencias básicamente, con diferencias de días de preparación y fueron analizadas para diferentes probetas cilíndricas, no se realizaron pruebas de vigas.

\section{ANÁLISIS Y COSTOS}

Del análisis de precios unitarios del hormigón y del proceso de la molienda de los neumáticos reciclados se puede apreciar que el precio de un $\mathrm{m}^{3}$ de árido fino producido por la molienda del neumático reciclado es elevado (121,74 dólares) comparado con la arena gruesa (22 dólares), sin embargo al ser un sustituto parcial de sólo un $10 \%$ de la arena gruesa y está a su vez representa un $10 \%$ en el valor del $\mathrm{m}^{3}$ de hormigón de $210 \mathrm{~kg} / \mathrm{cm} 2$, se observa que la diferencia del precio entre el hormigón sin molienda y con la misma es de 8,37 dólares lo que representa de solo un $3,6 \%$ de aumento en el costo del hormigón.

\section{CONCLUSIONES}

Los diseños son aceptables con la inclusión de caucho triturado como sustituto parcial, tanto del agregado grueso como del agregado fino en la elaboración de hormigones de resistencias a la compresión de $210 \mathrm{Kg} / \mathrm{cm}^{2}$.

Que el diseño de hormigón con un $10 \%$ de caucho triturado incorporado como sustituto parcial del agregado fino, dio como resultado ser el más apropiado para fines constructivos ya que alcanzó una eficiencia del $107 \%$ del patrón, de tal manera que se justifica su uso en las diferentes obras de ingeniería.

Que el hormigón elaborado con el 10\% de caucho triturado como sustituto parcial del agregado grueso, alcanza resistencias de hasta el $96 \%$ del patrón por lo que es recomendable usarlo ya que cumple con las normas ecuatorianas de construcción. 
De los resultados de los ensayos realizados se pudo evaluar que la resistencia máxima de las probetas para el ensayo de compresión se puede alcanzar a una edad de 90 días para cilindros con $10 \%$ de caucho triturado incorporado como sustituto parcial del agregado grueso.

Si bien es cierto que la molienda del neumático cuesta 121,74 dólares, actualmente, y es un precio elevado, este se justifica con lo que cuesta tratar una tonelada de desecho que cuesta alrededor de 130 dólares por lo que resulta conveniente utilizar la molienda del neumático como sustituto parcial del árido fino y grueso en el hormigón.

\section{RECOMENDACIONES}

Como parte de esta investigación se recomienda avanzar los diseños con vigas que pudieran resultar favorables para diseños de pavimentos rígidos en carreteras, en esta investigación no se ha completado la serie de ensayos.

Realizar diseños de hormigones con resistencias a la compresión del $140 \mathrm{~kg} / \mathrm{cm}^{2}$ y $180 \mathrm{~kg} / \mathrm{cm}^{2}$ usando el caucho triturado reciclado como sustituto parcial del agregado grueso y fino.

En promedio se obtiene $2.4 \mathrm{~kg}$ de caucho triturado por llanta, una vez separado el alambre y la cara de apoyo a la calzada de la llanta. Es decir, solo se utilizó el caucho ubicado en las caras laterales de las llantas.

Dado que se utiliza las caras laterales de los neumáticos para obtener materia prima para los diseños de hormigón, y al ser llantas de desecho, el área que ocupan los residuos en los botaderos, ya sean improvisados o municipales, se reduce en más del $50 \%$ lo que ayuda al ornato y aseo local.

Esta investigación se basó exclusivamente en realizar los ensayos con cauchos reciclados de neumáticos usados obtenidos de una máquina de laboratorio, pero si se lo ejecutara de manera industrial se debe considerar otros rendimientos y con cortes de los neumáticos con los cercos de alambre, los mismos que pueden ser incorporados en los hormigones, aumentando la resistencia.

Lo que se ha demostrado con esta investigación es la gran ventaja de reducir los impactos ambientales de no botar a los basureros los neumáticos, pudiendo ser utilizados como materia prima y de gran utilidad en las obras de Ingeniería Civil.

Crear centros de acopio en cabeceras cantonales y comenzar a dar uso de esta materia prima de gran utilidad en las obras de infraestructura civil.

\section{BIBLIOGRAFÍA:}

- [1] Revista Científica y Tecnológica UPSE, VOL. 2, NÚM. 3 (2015).

- [2] Carrasco, Ma. Fernanda (2013). Tecnología del Hormigón, Universidad Tecnológica Nacional, Colombia.

- [3] Romo, Marcelo (2008). Temas de Hormigón Armado, ESPE, Ecuador.

- [4] CD Continental General Tire Mantenimiento de neumáticos 2008

- [5] Neville,

- [6] Romo, Marcelo.

- [7] ACI 318S-08, 2008.American Concrete Institute, Requisitos de reglamento para concreto estructural y comentarios.

- [8] Instituto ecuatoriano del cemento y del concreto, (2009). Notas Técnicas, Control de Calidad en el hormigón, Control por resistencia, Parte I. Primera Edición.

- [9] Polanco, Abraham 2010, Manual de Prácticas de Laboratorio de Concreto, Universidad Autónoma de ChihuahuaFacultad de Ingeniería.

- [10] Instituto Ecuatoriano de Normalización, (2010). Hormigón de Cemento Hidráulico. Determinación de la Resistencia a la compresión de especímenes cilíndricos de hormigón de cemento hidráulico. (NTE-INEN 2554:2011). Primera Edición, Quito, Ecuador, 8 pag..

- [11] Carrasco, María Fernanda (2009). Apuntes de la Asignatura Tecnología del Hormigón. Facultad de Ciencias Exactas, Ingeniería y Agrimensura, Universidad Tecnológica Nacional, Santa Fe, Argentina.

\section{Linkografía:}

- $\quad$ http://publiespe.espe.edu.ec/academicas/ho rmigon/hormigon02-a.htm

- http://www6.uniovi.es/usr/fblanco/Tema8. Materiales.Construccion.Hormigon.pdf 
- http://disensa.com/librogrande/libro/libro_ grande.html

- $\quad$ file:///C:/Users/usuario/Downloads/Unidad 3_ROCAS_Y_AGREGADOS_PARA_H ORMIGONES.pdf (

- http://es.slideshare.net/yahil350/ensayode-materiales-13447018\#

- http://www.construmatica.com/construpedi a/Hormig\%C3\%B3n_Endurecido

- http://civilgeeks.com/2011/12/11/propieda des-principales-del-concreto/

- http://ingevil.blogspot.com/2008/10/ensay o-compresin-de-cilindros-de.html

- http://www.ingenierocivilinfo.com/2011/0 6/modulo-de-elasticidad-delhormigon.html

- https://upcommons.upc.edu/pfc/bitstream/ 2099.1/3324/8/55872-8.pdf

- http://notasdehormigonarmado.blogspot.co m/2011/04/8-metodo-de-ensayo-traccionindirecta.html

- https://upcommons.upc.edu/pfc/bitstream/ 2099.1/3252/9/50777-9.pdf

- http://www6.uniovi.es/usr/fblanco/Medida ConsistenciaHORMIGON.ConoABRAMS .pdf 